\title{
Echinochloa crus-galli genome analysis provides insight into its adaptation and invasiveness as a weed
}

Longbiao Guo (1) 1, Jie Qiu (1) 2, Chuyu Ye², Gulei Jin³, Lingfeng Mao ${ }^{2}$, Haiqiang Zhang ${ }^{2}$, Xuefang Yang ${ }^{4}$, Qiong Peng ${ }^{5}$, Yingying Wang ${ }^{2}$, Lei Jia ${ }^{2}$, Zhangxiang Lin² ${ }^{2}$ Gengmi Li ${ }^{1}$, Fei Fu ${ }^{2}$, Chen Liu ${ }^{2}$, Li Chen², Enhui Shen², Weidi Wang ${ }^{2}$, Qinjie $\mathrm{Chu}^{2}$, Dongya $\mathrm{Wu}^{2}$, Sanling $\mathrm{Wu}^{6}$, Chenyang Xia ${ }^{7}$, Yongfei Zhang ${ }^{5}$, Xiaomao Zhou ${ }^{5}$, Lifeng Wang ${ }^{5}$, Lamei Wu ${ }^{5}$, Weijie Song ${ }^{3}$, Yunfei Wang ${ }^{8}$, Qingyao Shu ${ }^{2}{ }^{2}$, Daisuke Aoki ${ }^{9}$, Emi Yumoto ${ }^{10}$, Takao Yokota ${ }^{10}$, Koji Miyamoto (10 ${ }^{10}$, Kazunori Okada11, Do-Soon Kim ${ }^{12}$, Daguang Cai (1) ${ }^{13}$, Chulong Zhang (i) ${ }^{7}$, Yonggen Lou7, Qian Qian', Hirofumi Yamaguchi ${ }^{14}$, Hisakazu Yamane ${ }^{10}$, Chui-Hua Kong ${ }^{4}$, Michael P. Timko ${ }^{15}$, Lianyang $\mathrm{Bai}^{5}$ \& Longjiang Fan ${ }^{2}$

Barnyardgrass (Echinochloa crus-galli) is a pernicious weed in agricultural fields worldwide. The molecular mechanisms underlying its success in the absence of human intervention are presently unknown. Here we report a draft genome sequence of the hexaploid species E. crus-galli, i.e., a $1.27 \mathrm{~Gb}$ assembly representing $90.7 \%$ of the predicted genome size. An extremely large repertoire of genes encoding cytochrome P450 monooxygenases and glutathione S-transferases associated with detoxification are found. Two gene clusters involved in the biosynthesis of an allelochemical 2,4-dihydroxy-7-methoxy-1,4-benzoxazin-3-one (DIMBOA) and a phytoalexin momilactone $A$ are found in the E. crus-galli genome, respectively. The allelochemical DIMBOA gene cluster is activated in response to co-cultivation with rice, while the phytoalexin momilactone A gene cluster specifically to infection by pathogenic Pyricularia oryzae. Our results provide a new understanding of the molecular mechanisms underlying the extreme adaptation of the weed.

\footnotetext{
${ }^{1}$ China National Rice Research Institute, Chinese Academy of Agricultural Sciences, Hangzhou 310006, China. ${ }^{2}$ Institute of Crop Science and Institute of Bioinformatics, Zhejiang University, Hangzhou 310058, China. ${ }^{3}$ Guhe Information, Hangzhou 310058, China. ${ }^{4}$ College of Resources and Environmental Sciences, China Agricultural University, Beijing 100193, China. ${ }^{5}$ Hunan Weed Science Key Laboratory, Hunan Academy of Agriculture Science, Changsha 410125, China. ${ }^{6}$ Analysis Center of Agrobiology and Environmental Sciences, Faculty of Agriculture, Life and Environmental Sciences, , Zhejiang University, Hangzhou 310058, China. ${ }^{7}$ State Key Laboratory of Rice Biology, Institute of Biotechnology, Zhejiang University, Hangzhou 310058, China. ${ }^{8}$ Zhejiang Sheng Ting Biotechnology Co., Ltd., Taizhou 318020, China. ${ }^{9}$ Department of Bioscience, Faculty of Biotechnology, Fukui Prefectural University, Fukui 910-1195, Japan. ${ }^{10}$ Department of Biosciences, Teikyo University, Tochigi 320-8551, Japan. ${ }^{11}$ Biotechnology Research Center, The University of Tokyo, Tokyo 113-8657, Japan. ${ }^{12}$ Department of Plant Science, Institute of Agriculture and Life Sciences, College of Agriculture and Life Sciences, Seoul National University, Seoul 151-921, Korea. ${ }^{13}$ Department of Molecular Phytopathology and Biotechnology, Christian-Albrechts-University of Kiel, Kiel D-24118, Germany. ${ }^{14}$ Faculty of Agriculture, Tokyo University of Agriculture, Kanagawa 243-0034, Japan. ${ }^{15}$ Department of Biology, University of Virginia, Charlottesville, VA 22904, USA. Longbiao Guo and Jie Qiu contributed equally to this work. Correspondence and requests for materials should be addressed to L.B. (email: bailianyang2005@aliyun.com) or to L.F. (email: fanlj@zju.edu.cn)
} 
gronomically important weeds cause tremendous worldwide losses of crop yields estimated at over $\$ 95$ billion annually according to United Nations Food and Agriculture Organization ${ }^{1}$. Within the short period of agricultural history, weedy plants have exploited the human-mediated environment of farmers' fields by undergoing rapid adaptive evolution that allowed them to proliferate and escape eradication using weediness traits such as rapid growth rates, prolonged dormancy, ease of dispersal, herbicide resistance, etc ${ }^{2}$. Thus, weeds have evolved to survive in agroecosystems under natural and unintentionally human-mediated selection ${ }^{3}$, leading to a distinct evolutionary state that has proceeded in parallel to crop domestication $^{2}$. Therefore, agricultural weeds are ideal models for the study of environmental adaption of plants from an evolutionary perspective. Beyond purely academic interests, uncovering how weeds evolve is crucial for their management and protection of the global food supply.

In contrast to numerous food and forage crops where the genomes of many important domesticated forms and their wild relatives have been sequenced leading to a better understanding of the genetic basis of many important agronomic traits ${ }^{4}$, little genomic scale analysis has taken place on agricultural weed species despite their economic significance. In fact, among the important agricultural weeds known, only the dicot horseweed (Conyza canadensis) has had its whole genome sequenced thus far ${ }^{5}$.

The genus Echinochloa (Poaceae) or barnyardgrass includes approximately 250 annual and perennial species ${ }^{6}$. Systematic frequency assessments for rice weeds based on phytosociological studies showed that Echinochloa species are the most frequent weeds associated with rice cultivation globally ${ }^{7}$. Among Echinochloa species, E. crus-galli (annual, $2 n=6 x=54$ ) is the most prevalent species ${ }^{8}, 9$, followed by Echinochloa colona and the tetraploid Echinochloa oryzicola ${ }^{7}$. While conscious artificial selection for useful agronomical traits drove changes in early rice species during domestication, wild barnyardgrass growing in the same paddy fields was driven by natural and unintentional selections to evolve adaptive and competitive characteristics to evade removal from rice fields. For example, E. crus-galli in paddy fields morphologically resembles rice (Oryza sativa) at the seedling stage, which increase the difficulty of recognition during manual weeding ${ }^{10}$. More recently, E. crus-galli acquired or evolved target-site and non-target-site herbicide resistance making it among the most pernicious herbicide resistant weeds of the world $^{11}$. Worldwide losses of yield in rice due to E. crus-galli competition are estimated to be about $35 \%^{12}$.

Allelopathy, or the ability of one plant to suppress the growth of another nearby plant through the release of chemical compounds (i.e., allelochemicals) in the rhizosphere is one of the most important weediness features. Allelopathy plays an important role in natural and agricultural ecosystems, particularly in crop ecosystems ${ }^{13}$ and has received much attention in recent years because it could potentially serve as an alternative weed management strategy in crop production without environmental $\operatorname{cost}^{14}$. Many field and greenhouse studies have demonstrated the allelopathic potential of rice cultivars on barnyardgrass ${ }^{14}$.

A number of plant secondary metabolites have been suggested to serve as allelochemicals and molecular genetic studies have shown that gene clusters are often responsible for the biosynthesis of the allelopathic compounds ${ }^{15}$. The first allelochemical gene cluster intensively studied was that involved in the formation of 2,4-dihydroxy-7-methoxy-1,4-benzoxazin-3-one (DIMBOA), a protective and allelopathic defense compound against weeds, present in maize (Zea mays) ${ }^{16}$. The maize DIMBOA gene cluster is located on Chromosome 4 and consists of eight genes (BX1 to $B X 8)^{16}$. In rice, two gene clusters involved in the biosynthesis of the allelopathic diterpene phytoalexins momilactone $\mathrm{A}$ and phytocassane have been identified on Chromosomes 4 and 2, respectively ${ }^{17,18}$. These diterpene phytoalexins can significantly inhibit growth of E. crus-galli.

Considerable attention has been devoted to understand allelopathic interactions between crops and weeds, but most studies have focused on the genetic mechanisms of the impact of crops on weeds. Knowledge of the underlying basis for how weeds respond to the allelopathic challenge from crops and why weeds are dominant in crop fields without human intervention is limited. In this study, we generated a draft genome assembly for the hexaploid species E. crus-galli, the dominant weed in rice paddy fields, and analyzed the dynamic changes in transcriptome profiles of E. crus-galli growing alone and in response to the cocultivation with rice plants. In the genome, We have identified three copies of gene clusters involved in production of an allelochemical (DIMBOA) used by E. crus-galli against rice and one copy of a phytoalexin (momilactone A) gene cluster against blast disease $(P$. oryzae) in the paddy field environments.

\section{Results}

Genome assembly and annotation. The E. crus-galli line STB08, collected from rice paddy fields in the lower Yangtze River region of China, highly resembles cultivated rice in morphology (Supplementary Fig. 1), and has a chromosome number of $2 n=6 x=$ 54. A total of $207.4 \mathrm{~Gb}$ of sequence data were generated using the Illumina HiSeq 2000 system from STB08 genomic DNA libraries with fragment sizes varying between $160 \mathrm{bp}$ to $20 \mathrm{~Kb}$ (Table 1; Supplementary Table 1). In addition, the Pacbio RS II system was used to generate $32.9 \mathrm{~Gb}$ third-generation long reads, totally

Table 1 Summary of the genome assembly and annotation of E. crus-galli

Sequencing and assembly

Sequencing

Scaffold

Insert libraries

$160 \mathrm{bp}-20 \mathrm{~Kb}$

N50 size $(\mathrm{Mb}) \quad$ N90 size $(\mathrm{Kb})$

Genome annotation

Protein-coding gene

Non-coding RNA

Repetitive elements (\%)
1.80

Gene models

108,771

miRNA

785

LTR

21.9

Illumina
$148.2 \times$
N90 size (Kb)
453.89
Supported by homologs ${ }^{a}$
$74.2 \%$
tRNA
2306
LINEs\& SINEs
2.5

Pacbio RS II
$23.5 \times$
The longest $(\mathrm{Mb})$
11.70

Supported by EST and Swiss-prot etc. ${ }^{b}$ $85.17 \%$

rRNA

1890

DNA transposon

8.0
Total size (Gb)

240.3

Total non-N size (Gb)

1.27

Gene size (bp)

1,901

snoRNA

3378

Total

40.7

${ }^{a}$ Annotated gene sets of six sequenced plants (B. distachyon, O. sativa, S. bicolor, Z. mays, S. italic, and A. thaliana)

bswiss-prot, InterProt and $\mathrm{nr}$ databases are included 
a

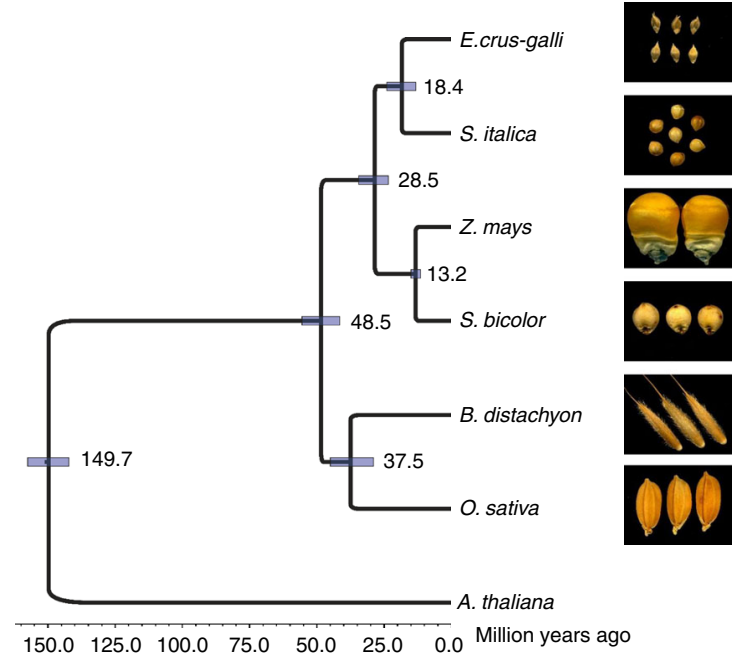

b

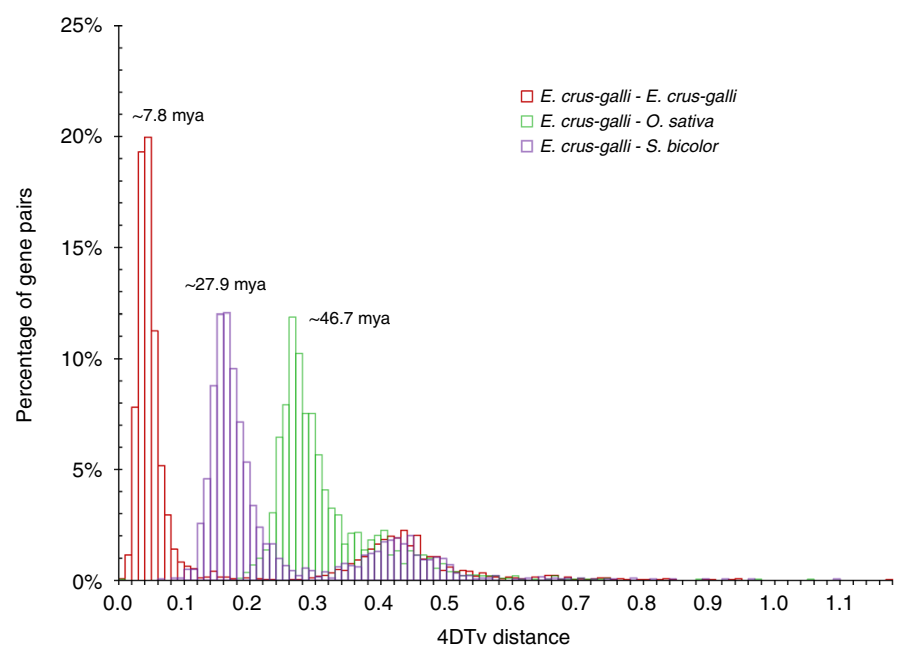

Fig. 1 Phylogenetics of E. crus-galli. a Single-copy gene based phylogenetic tree and divergence times of E. crus-galli and other grasses with Arabidopsis as an outgroup. b Distribution of the transversion rates at the four-fold degenerate sites ( $4 \mathrm{dTV}$ ) of paralogous gene pairs of $E$. crus-galli and other grasses. The times of E. crus-galli speciation and E. crus-galli-Sorghum and -Oryza divergence are given based on corresponding paralogous and orthologous peaks

representing $\sim 171 \times$ coverage of the E. crus-galli genome estimated to be $\sim 1.4 \mathrm{~Gb}$ in size based on the $K$-mer analysis and flow cytometry (Supplementary Fig. 2). De novo assembly yielded a draft genome of $1.27 \mathrm{~Gb}$, representing $90.7 \%$ of the E. crus-galli genome $(>1 \mathrm{~Kb})$, with a scaffold N50 length of $1.8 \mathrm{Mb}$. Five fosmid clones ( $>15 \mathrm{~Kb}$ ) were sequenced and compared with the assembly, and is confirm to be of good consistence (Supplementary Table 2). About $92.3 \%$ of the core eukaryotic genes (CEGs) could be completely aligned with the E. crus-galli gene set. We have also used BUSCO to judge the assembly of E. crus-galli, and found that the 'complete' percent is $95.5 \%$, which is comparable to that of S. bicolor (96.4\%) and S. italica (94.3\%) genome (Supplementary Table 3 ). In addition, we mapped 74 publicly available E. crus-galli EST sequences and 156,757 transcripts generated by this study to the assembled genome and observed high mapping and identity rates (Supplementary Table 3).

For gene annotation, transcriptomic data from the whole plant were generated by RNA-Seq (Supplementary Table 4). By integrating gene finding results from $a b$ initio, homology- and transcript-based approaches, we predicted 108,771 proteincoding in the E. crus-galli genome (Table 1). Of the 108,771 genes, $85 \%$ were supported by either the identification of homologues in other species or RNA-Seq data. In addition to protein-coding genes, 785 microRNAs (miRNAs) and other noncoding RNAs were also identified in the E. crus-galli genome (Table 1 and Supplementary Table 5). On the basis of homology searches and de novo methods, we identified a total of $514 \mathrm{Mb}$ of repetitive elements which represents $40.7 \%$ of the genomic assembly (Table 1). Among the repetitive sequences, long terminal retrotransposons (LTRs) were the most abundant, accounting for $21.9 \%$ of the assembly (Supplementary Table 6).

Using pairwise protein sequence comparisons, putative orthologs and paralogs are analyzed among E. crus-galli and five other grass family members, including $B$. distachyon, $O$. sativa, $S$. bicolor, Z. mays, and S. italica. We found 26,679 gene families (containing a total of 72,363 genes) in the E. crus-galli genome, of which 6,789 families containing 20,739 genes appear to be Echinochloa-specific. On the basis of the orthologous single-copy genes, the Oryza- and Sorghum-Echinochloa divergence times were estimated to be ca. 48.5 and 28.5 million years ago (Mya), respectively (Fig. 1a).
E. crus-galli is an allohexaploid and therefore should be associated with ancient polyploidization events. We mapped E. crus-galli scaffolds to the diploid sorghum and foxtail millet (S. italica) genomes, and found that all chromosomes of sorghum and foxtail millet had three copies of E. crus-galli sequences aligned to them (Supplementary Fig. 3), consistent with E. crusgalli being a hexaploid. We calculated the transversion rate at the four-fold degenerate sites $(4 \mathrm{dTv})$ of paralogous and orthologous gene pairs in the E. crus-galli genome. The orthologous peaks of Echinochloa-Oryza and -Sorghum had a corresponding divergence of $4 \mathrm{dTv} \approx 0.27$ and 0.16 and dated to 46.7 and $27.9 \mathrm{Mya}$, respectively (Fig. 1b), which are consistent with the estimations based on the phylogenetic tree (Fig. 1a). The paralogous gene pairs based on syntenic blocks in the E. crus-galli genome showed one obvious large paralogous peak with $4 \mathrm{dTv} \approx 0.042$ dated the polyploidization event(s) for E. crus-galli speciation at $\sim 7.8 \mathrm{Mya}$ (Fig. 1b).

Gene families associated with detoxification. Gene families such as cytochrome P450 monooxygenase (CYP450) and glutathione S-transferase (GST) are commonly associated with the detoxification of allelopathic compounds or non-target-site resistance to synthetic herbicides in weeds ${ }^{19}$. Additionally, some members of the CYP450 family are often involved in the secondary metabolism biosynthesis ${ }^{15}$. In the E. crus-galli genome, a total of 917 CYP450 and 277 GST genes were identified, which is significantly higher than the numbers found in the genomes of the other five grasses (O. sativa, B. distachyon, S. bicolor, S. italica, $Z$. mays) used for comparison in this study and in Arabidopsis (249354 CYP450 and 65-107 GST in a single genome). Enrichment analyses of Pfam domains showed that genes with the Pfam PF00067 (Cytochrome P450) and PF13417 (Glutathione Stransferase, N-terminal domain) are over-presented in E. crusgalli using other five grass species as background (PF00067: $P$ value $=3.52 \mathrm{E}-9 ; \mathrm{PF} 13417: P$-value $=1.12 \mathrm{E}-3$ both by hypergeometric test). Phylogenetic analysis indicates that the E. crus-galli genome contains more diverse E. crus-galli-specific branches in the two gene families relative to rice (CYP450 as an example shown in Supplementary Fig. 4). In addition, we investigated divergence times of CYP450 and GST genes in E. crus-galli and rice, respectively. A significantly large peak was observed at the 
range of very low amino acid substitution rates for CYP450 and GST genes in E. crus-galli, indicating a recent expansion which was likely caused by polyploidization events generating a large number of CYP450 and GST genes in E. crus-galli (Fig. 2).
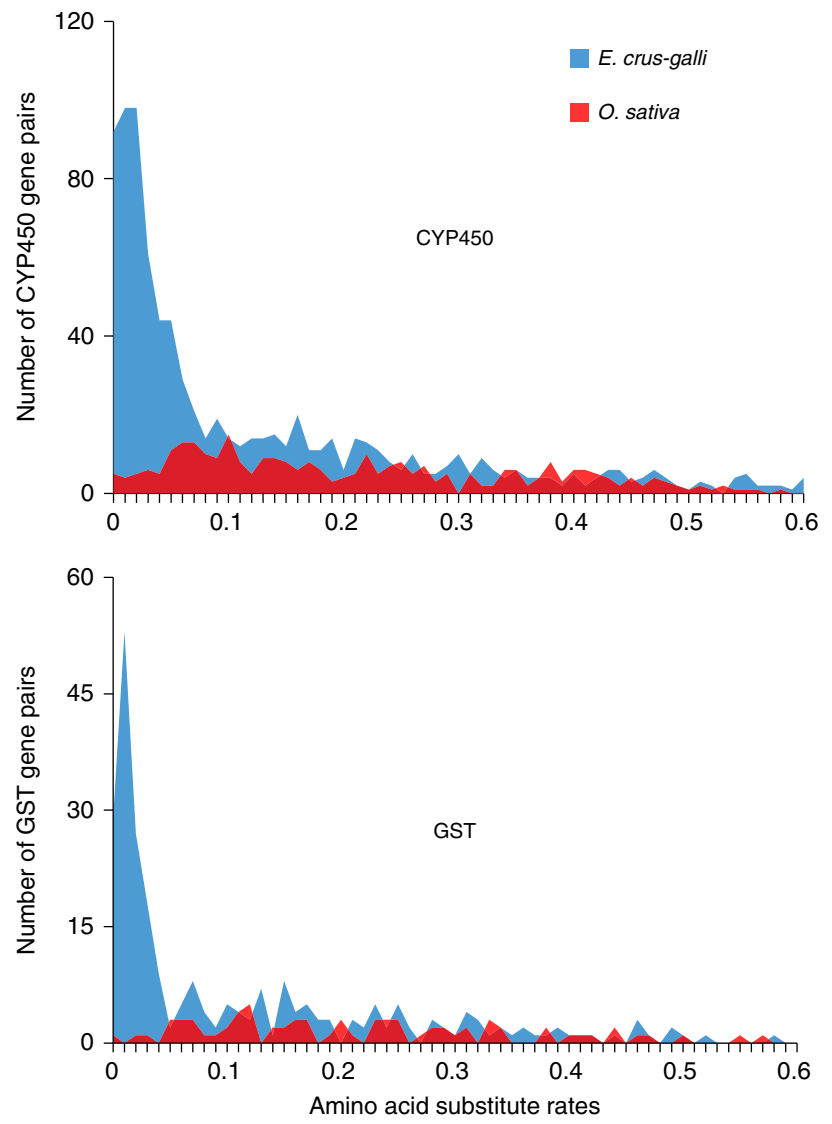

Fig. 2 Gene families associated with detoxification in E. crus-galli. Distribution of amino acid substitute rates of CYP450 and GST genes in E. crus-galli and O. sativa indicating a recent expansion of them in E. crus-galli
Transcriptomic profiles of E. crus-galli and rice plants involved in allelopathic interactions show that many genes from the two gene families are up-regulated (see below). These results suggest that the large repertoire of the CYP450 and GST genes may provide barnyardgrass enhanced detoxification ability under competition with nearby crop plants.

Transcriptomic profiles of $\boldsymbol{E}$. crus-galli allelopathy against rice. To identify candidate genes involved in allelopathy of barnyardgrass against rice, the E. crus-galli line STB08 and a rice cultivar PI312777 (known to have a high allelopathic potential with barnyardgrass ${ }^{20}$ ) were grown separately (mono-cultured) or cocultured (Supplementary Fig. 5). Transcriptome analysis was performed on the barnyardgrass plants following $3 \mathrm{~h}$ of cultivation. Using transcriptomes of mono-cultured plants as reference, a total of 4945 differentially expressed genes (DEGs), including 2534 up-regulated and 2411 down-regulated genes, were identified. Pathway enrichment analyses using Mapman software suggested that the significantly down-regulated genes in co-cultivated barnyardgrass are involved in photosynthesis and tetrapyrrole synthesis, suggesting rice plants growing with the barnyardgrass may have imposed an allelopathic effect on the weed by inhibiting its photosynthetic gene expression (Fig. 3a). In contrast, pathways associated with 'cytochrome P450', 'brassinosteroid hormone metabolism', 'phenylpropanoid metabolism' were enriched and the majority of the DEGs were up-regulated in the co-cultured barnyardgrass (Fig. 3b; Supplementary Data 1; Supplementary Fig. 6). Phenylpropanoid metabolism in particular generates an enormous array of secondary metabolites ${ }^{21}$. Brassinosteroids are phytohormones that have significant growth-promoting activity in plants and are involved in response to biotic and abiotic stresses $^{22}$. The identified up-regulated DEGs in these enriched pathways may confer critical response pathways allowing barnyardgrass to protect itself against rice during their allelopathic interactions.

The gene clusters for biosynthesis of DIMBOA in E. crus-galli. The gene cluster for DIMBOA production has been described only in maize so far, although the presence of DIMBOA has been reported in other upland plants (such as wheat and rye) ${ }^{23}$. Three a
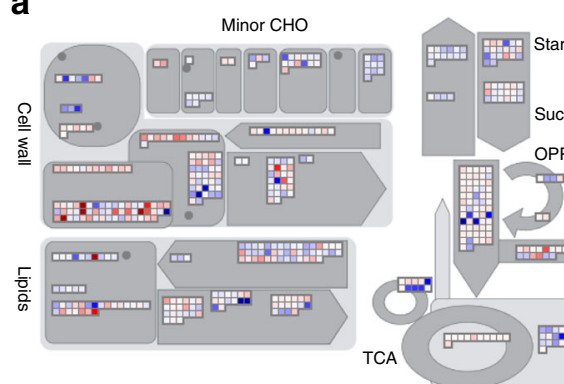

Aascorbate, Glutathione Light
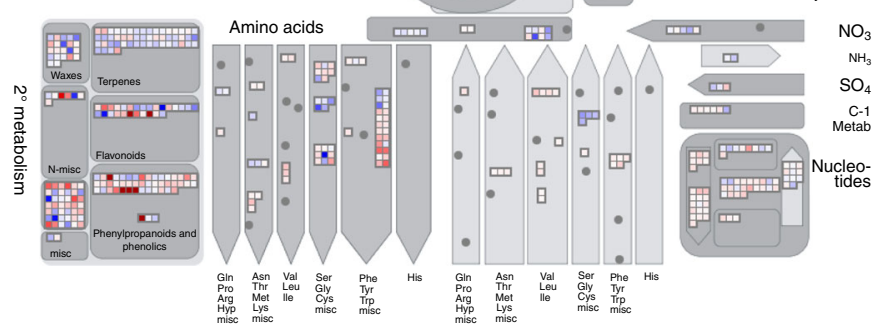

b

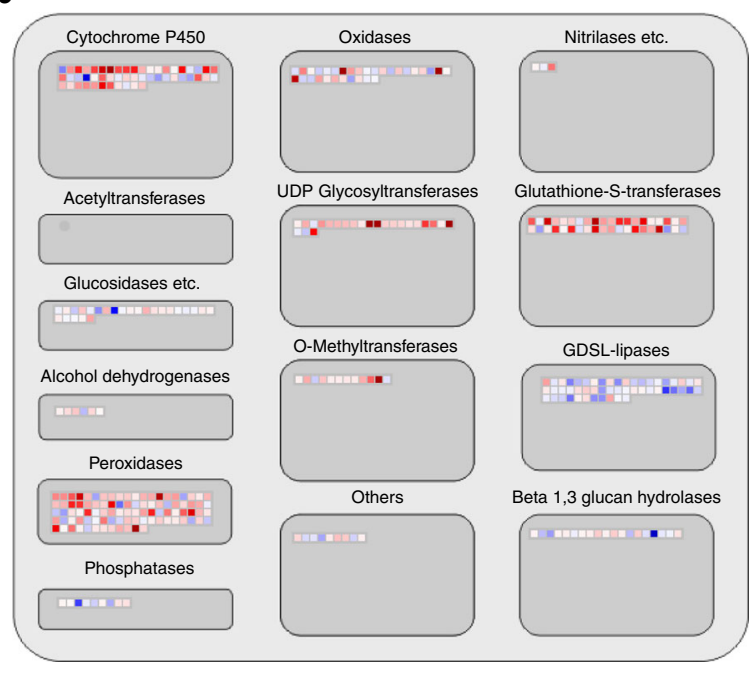

Fig. 3 The allelopathic transcriptomic profiles of barnyardgrass against rice. a An overview for diverse pathways and $\mathbf{b}$ transcriptomic profile for large enzyme families visualized by Mapman. Color intensity corresponds to the expression fold change at log2 scale (red: up-regulated, blue: down-regulated) 
a

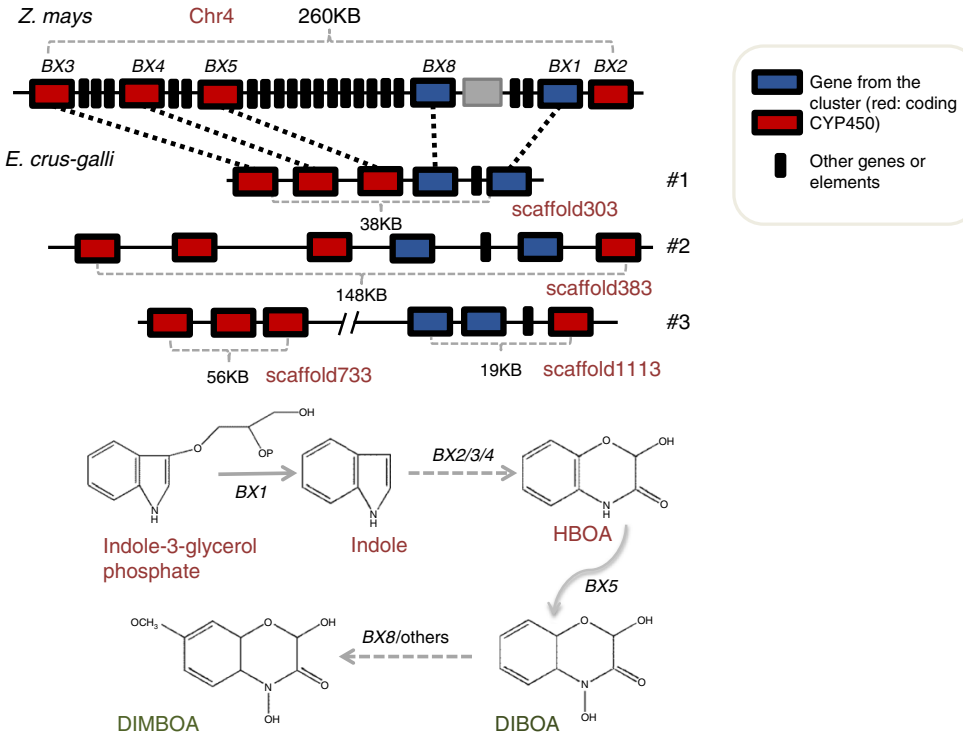

C

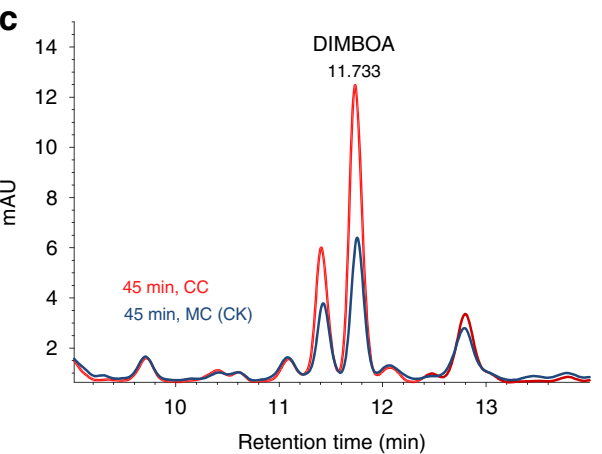

b
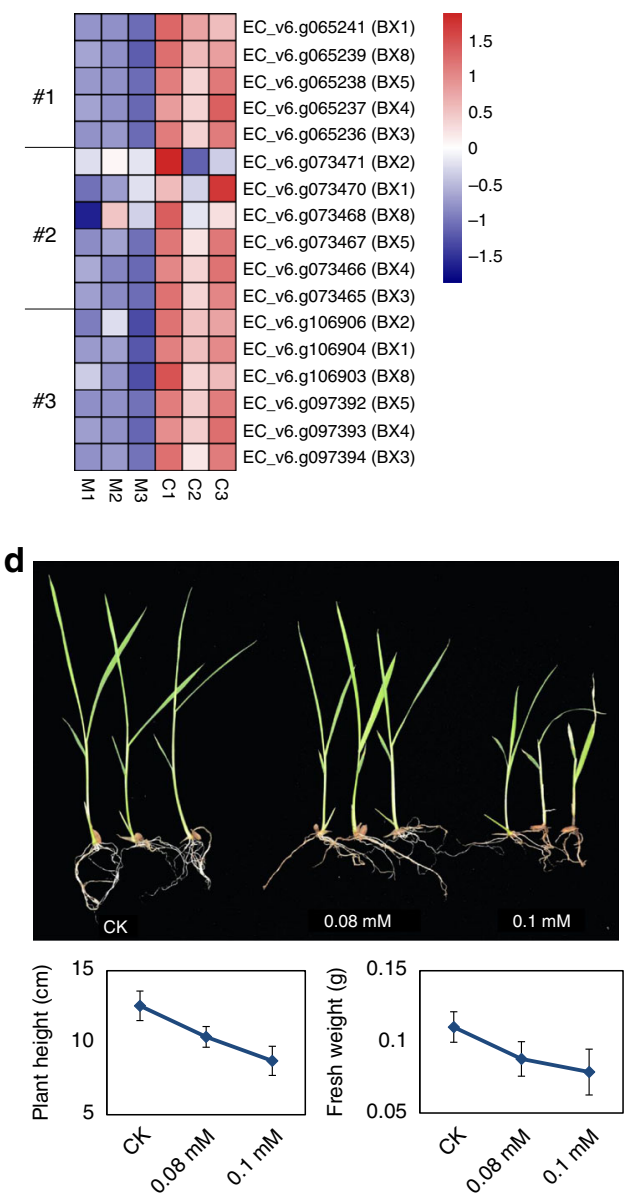

Fig. 4 Characterization of biosynthetic gene clusters for DIMBOA in E. crus-galli. a Gene cluster for DIMBOA. Top, genomic synteny of the gene clusters for DIMBOA between barnyardgrass and maize. Bottom, the biosynthetic pathway of DIMBOA. $\mathbf{b}$ Expression changes of genes in the three copies of DIMBOA gene cluster in barnyardgrass during co-culture. Expression values were scaled for each gene. ' $M$ ' refers to mono-cultured experiments, and ' $C$ ' refers to experiments co-cultured with rice. c DIMBOA quantification in barnyardgrass. Left, selected HPLC ion chromatograms of root exudate extracts of barnyardgrass seedlings from mono-cultured (MC, as control) or co-cultured with rice (CC). Right, comparison of the DIMBOA concentrations in E. crusgalli from mono- and co-cultivated conditions at the specified time points. $\mathbf{d}$ Effect of DIMBOA treatment on the growth of rice seedlings. Top, phenotype of rice seedlings after one week treatment of 0.08 and $0.1 \mathrm{mM} \mathrm{DIMBOA}$. Bottom, effects of DIMBOA treatment on plant height and fresh weight of rice seedlings

copies of the DIMBOA gene cluster are found in the E. crus-galli STB08 genome each with perfect synteny with the maize genomic segment containing $B X 1-5$ and $B X 8$ (Fig. 4a). Our transcriptome data indicated that almost all copies of these genes (with the exception of BX2 and BX8 in copy 2) in E. crus-galli were significantly up-regulated in barnyardgrass co-cultured with rice compared to mono-cultured STB08 (Fig. 4b; Supplementary Data 2). To confirm that the induced expression of the DIMBOA biosynthetic gene cluster of barnyardgrass in response to cocultivation with rice resulted in increased allelochemical production, we quantified the DIMBOA content in barnyardgrass. As shown in Fig. 4c, DIMBOA was significantly increased in barnyardgrass co-cultured with rice at all three time points ( $45 \mathrm{~min}$, 1.5 and $3 \mathrm{~h}$ ). As expected, no DIMBOA was detected in rice control plants.

Analysis of the effects of DIMBOA on rice growth showed that after DIMBOA treatment, rice plants showed a significant reduction in plant height and fresh weight. The inhibitory effects of DIMBOA were positively correlated DIMBOA concentration (Fig. 4d). Cumulatively these data suggest that the biosynthesis of DIMBOA by barnyardgrass is likely a significant mechanism in its competition with rice in the paddy field.
It is assumed that E. crus-galli (hexaploid) arises from the hybridization between tetraploid E. oryzicola (paternal donor) and an unknown diploid species (maternal donor) ${ }^{8}$. To determine the origins for the three copies of the DIMBOA gene clusters in $E$. crus-galli genome, we sequenced an E. oryzicola line (ZJU2, an estimated genome size of $\sim 1 \mathrm{~Gb}$ ) to $\sim 30 \times$ coverage (Supplementary Table 1 and Supplementary Fig. 7). Mapping results showed that scaffolds for two of the DIMBOA clusters (cluster copy 2 and 3) were well covered by the E. oryzicola reads, whereas the scaffold for cluster copy 1 could barely be mapped (Supplementary Fig. 7a-d). These results indicate that the three copies of the DIMBOA cluster in the hexaploid species E. crusgalli were likely generated from a hybridization event, during which two DIMBOA copies were contributed by the tetraploid $E$. oryzicola while the unknown diploid species ancestor contribute to another DIMBOA copy.

The gene cluster for biosynthesis of momilactone $A$ in E. crusgalli. Interestingly, we also found a syntenic gene cluster in barnyardgrass corresponding to the gene cluster responsible for momilactone A biosynthesis in rice (Fig. 5a). Four adjoining 

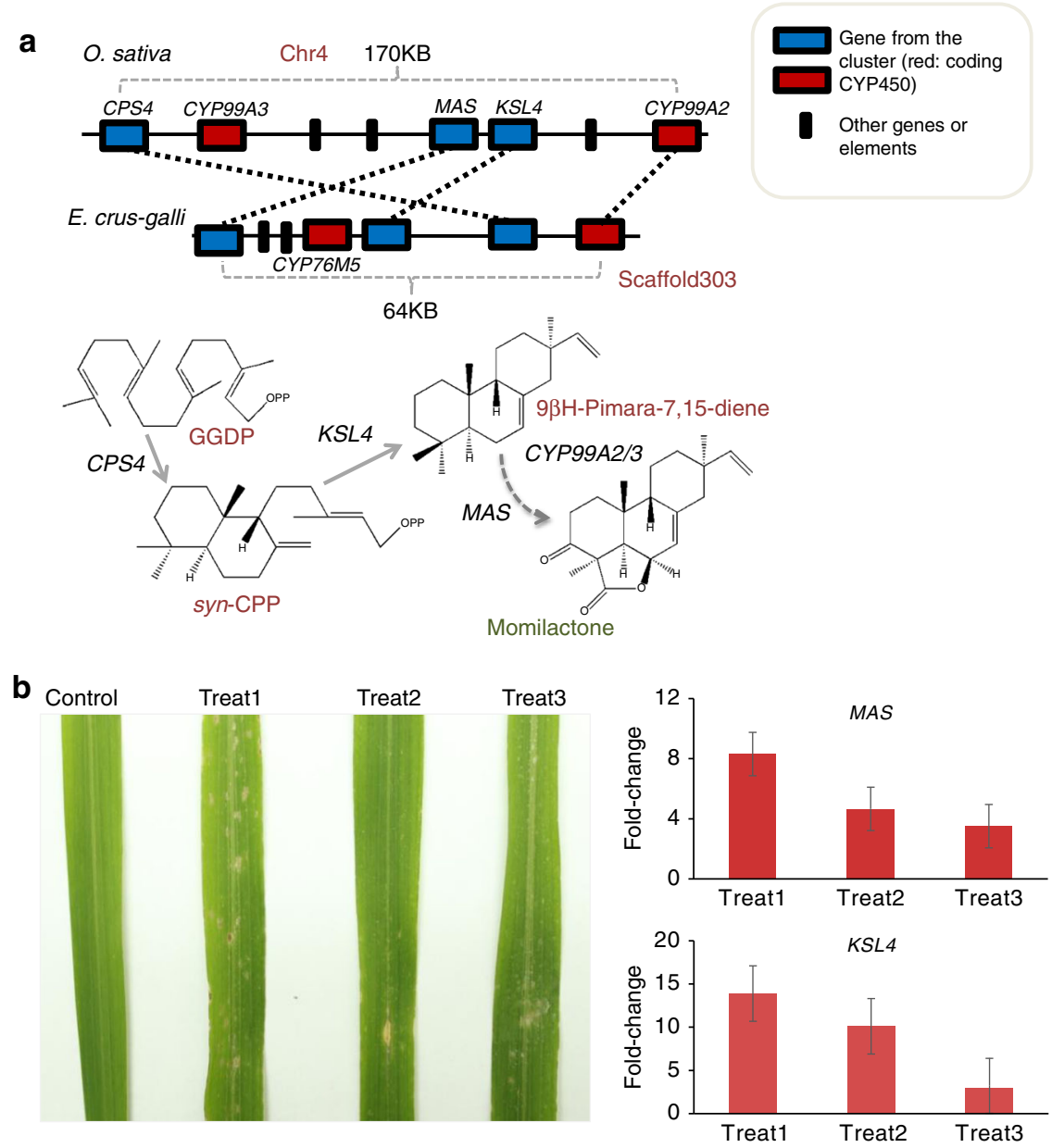

Fig. 5 Characterization of the biosynthetic gene cluster for momilactone A in E. crus-galli. a Gene clusters responsible for biosynthesis of momilatone A in rice and barnyardgrass. Top, genomic synteny of the gene clusters for momilatone A between barnyardgrass and rice. Bottom, the biosynthetic pathway of momilatone A. Genomic positions of the gene clusters were adopted from Boycheva et al. ${ }^{15}$. b Phenotypes of leaves and expression changes of genes in the momilactone A cluster of $E$. crus-galli under P. oryzae infection. Left, phenotypes for three leaves under $P$. oryzae infection and one as control. Right, gene expression changes for the three infected leaves, three technical replicates were performed for each leave. Expression value of genes without $P$. oryzae infection (control) was normalized to be one

genes in barnyardgrass were orthologous to four members of the gene cluster in rice despite some changes of gene order in the two genomes (Fig. 5a). However, the four genes in the E. crus-galli momilactone A biosynthesis gene cluster (only one copy found in the E. crus-galli genome) did not show any expression based on transcriptome analysis during barnyardgrass-rice co-cultivation. It has been previously reported that momilactones functions as both allelopathic and antimicrobial chemicals of rice against weeds and fungal pathogens, but can only exert little inhibition effect on the growth of seedlings of itself $^{24}$. Besides, it has also been reported that momilactone $\mathrm{A}$ is significantly induced by blast $(P$. oryzae) infection of rice in paddy environments and serves as phytoalexin to enhance blast resistance in leaves ${ }^{25}$. Therefore we hypothesized that the momilactone A cluster in the E. crus-galli was also associated with fungal resistance through the antimicrobial activity of momilactone A in the paddy filed. To test this directly, we infected STB08 leaves with P. oryzae (Fig. 5b) and then examined the expression of the momilactone A synthase $(M A S)$ and kaurene synthase-like (KSL4) genes in the momilactone A cluster using qRT-PCR. Our data show that the expression for the MAS and KSL4 genes dramatically increased (in some cases exceeding 10 -fold increases) after infection (Fig. 5b), indicating that the momilactone A gene cluster of $E$. crus-galli is activated by fungal stress and contributes to resistance to blast infection in paddy environment.

As the momilactone A gene cluster was previously only found in Oryza species ${ }^{26}$, the identification of a momilactone gene cluster in E. crus-galli that is distantly related to that found in $O$. sativa raises questions as to its evolutionary origins. Therefore, we conducted a phylogenetic analysis of the genes in the momilactone cluster from E. crus-galli and those from other grasses. Interestingly, our analysis showed that the E. crus-galli KSL4, CPS4, and MAS first clustered with their orthologs from Oryza species and then other cereals (Supplementary Fig. 8; Detailed gene labels see Supplementary Data 3). This clustering seems in contrast with the fact that E. crus-galli belongs to tribe Paniceae of the subfamily Panicoideae and near S. italica and P. virgatum, while Oryza species belong to the subfamily Oryzoideae (Fig. 1a; Supplementary Fig. 8a). The tree for CYP450 gene (CYP99A2) in the momilactone A cluster is consistent with the species phylogeny (i.e., they clustered with their orthologs in Setarial Panicum first) (Supplementary Fig. 8).

We also checked whether the genomic segment harboring the momilactone A cluster in E. crus-galli could be mapped by reads from E. oryzicola. Almost no reads could be mapped on the genes for the cluster (Supplementary Fig. 7e), suggesting that the 
momilactone A gene cluster in the E. crus-galli genome was not contributed by its paternal donor E. oryzicola.

\section{Discussion}

We have generated the draft genome of the hexaploid species, $E$. crus-galli, one of the most notorious weeds known in modern crop fields. Our results provide new insights into the adaptive molecular mechanisms for their survival and invasiveness in the paddy field. Our data first shows that (1) E. crus-galli has evolved a large repertoire of detoxification-related genes (e.g., CYP450 and GST) and many of them are responsive during allelopathic interactions with rice; (2) E. crus-galli growing in paddy fields evolved multiple copies of the DIMBOA gene cluster which it uses in allelopathic interactions against rice and (3) also employs a momilactone A gene cluster against pathogenic fungi (and perhaps other biotic stresses) in the paddy field.

For polyploid species, sub-genomic sequence similarity is one of the challenges for genome assembly ${ }^{27}$, as it may be difficult to distinguish two or more similar sub-genomes in a species, which could possibly introduce mis-assembly and can influence following utility of the draft genome. Luckily, it seems that the divergence level among sub-genomes of allopolyploid species (e.g., tobacco ${ }^{28}$, cotton ${ }^{29}$, rapeseed ${ }^{30}$, and also E. crus-galli in this case) is high enough, and classical short sequencing data based assembly tools, such as SOAPdenovo and ALLPATHS-LG, can assemble their genomes well to a comparable assembly statistics (such as scaffold N50) to that of many diploid genomes. Currently, third generation sequencing (TGS) technologies have been comprehensively used in genome assembly. However, the polyploidy genome features could pose more challenges for utilization these data. It should be noted that the average sequencing error rate of TGS is still very high $(\sim 15 \%)$, which could be similar or even higher than the sequence differences between the subgenomes of polyploidy species. Therefore, pre-correction step for TGS reads are quite necessary for using these data. In addition, we found that in our case, only less than $6 \mathrm{~Gb}$ corrected Pacbio data (efficiency $<20 \%$ ) is left after correction by Canu, which may indicate that more raw data are needed for polyploidy genome sequencing projects.

Biosynthetic gene clusters responsible for known allelopathic compounds and phytoalexin (DIMBOA and momilactones) were found in the E. crus-galli genome, which have been identified in crops (such as maize and rice). Several benefits of gene clusters include improved efficiency in functionality, heredity, and survival compared with non-clustered genes functioning in the same pathway ${ }^{15,31}$. These evolved functional gene clusters may imply one of the critical genetic bases for rapid environmental adaptability of the weed. For example, our results demonstrated that DIMBOA is an essential allelochemical employed by barnyardgrass to inhibit rice growth in paddy fields. E. crus-galli have evolved to form multiple copies of DIMBOA gene cluster, which should play a critical role in competition with rice and its invasiveness in paddy fields. Further, as to implications for rice breeding, it is crucial to find genes or loci responsible for DIM$\mathrm{BOA}$ resistance in rice genome, and rice cultivars with improved DIMBOA resistance may be produced through molecular breeding in the future.

The syntenic gene cluster for momilactone A has previously only been found in the rice genome. On the basis our transcriptome profiling analysis of baryardgrass and rice plants under co-cultivation, it does not appear that the momilactone A gene cluster in E. crus-galli is activated during these interactions. While the exact role(s) of the momilactone A cluster in E. crus-galli still requires further investigations, it appears that it may be involved in regulating biotic stress, primarily in response to infection of $P$. oryzae, a significant constraint on growth in rice paddies. The evolved momilactone A gene cluster in E. crus-galli could enhance its adaptability in paddy environment.

In investigation of the evolutionary history of momilactone $\mathrm{A}$ cluster, we only found one copy of momilactone A gene cluster in E. crus-galli genome, and it appears that the tetraploid paternal species E. oryzicola does not harbor this cluster or the related genes. Several reasonable explanations can be put forth to account for this observation. First, the unknown diploid Echinochloa species itself equips the momilactone A cluster, and contribute the cluster to E. crus-galli during hybridization with E. oryzicola. Alternatively, the diploid species does not harbor the cluster but has the related genes. After hybridization, the cluster formed in the E. crus-galli genome with genomic rearrangements of genes (CPS4, KSL4, MAS, and CYP99A) involved in the biosynthetic pathway. The unknown diploid Echinochloa species material is crucial for better understanding of the evolutionary mystery of the momilactone A gene cluster, and the environmental adaptation of Echinochloa weeds. Our phylogenetic analyses showed that genes of E. crus-galli in the momilactone A gene cluster seems closer to O. sativa than other phylogenetically close species (e.g., B. distachyon, Z. latifolia or even O. brachyantha). The unexpected phylogenetic distributions suggested that complex processes (e.g., multiple gene duplications followed by different losses, or even horizontal gene transfer) could be involved in the evolution of the genes (CPS4, KSL4, MAS) and the formation of the momilactone gene cluster in E. crus-galli, which need further investigation in future.

\section{Methods}

Genome sequencing and assembly. The E. crus-galli line STB08, collected from rice paddy fields in the lower Yangtze River region of China $\left(30^{\circ} 17^{\prime} \mathrm{N}, 119^{\circ} 57^{\prime} \mathrm{E}\right)$ Genomic DNA was extracted from young leaves of E. crus-galli (STB08) plants using the CTAB method. RNase A and proteinase K were used to remove RNA and protein contamination, respectively. Paired-end and mate-pair Illumina genomic DNA libraries with different insertion sizes $(160 \mathrm{bp}-20 \mathrm{~Kb})$ were constructed following the manufacturer's instructions (Illumina, USA). The libraries were sequenced on an Illumina HiSeq 2000 system. Meanwhile, two Pacbio RS II P4-C2 libraries of STB08 were constructed following the manufacturer's instructions and 48 SMRT cells $(32.9 \mathrm{~Gb})$ were sequenced. Raw reads were processed by removing PCR duplicates, low-quality reads, adaptor sequences and contaminated reads with bacterial or viral origin. In addition, we used Lighter software ${ }^{32}$ to correct the reads from each library based on the $K$-mer frequency. The resulting clean reads were assembled into contigs and scaffolds using SOAPdenovo $2^{33}$ with the default settings. The assembled sequences were further scaffolded with OPERA-LG v2.0.5 ${ }^{34}$, and gaps within the scaffolds were filled using GapCloser v1.1 $1^{33}$. Redundans ${ }^{35}$ was used to filter redundant sequences due to heterozygosity. Raw Pacbio RS long reads were corrected by $\mathrm{Canu}^{36}$, and then were used to fill the gaps by PBjelly v14.1with the default settings ${ }^{37}$. Detailed assembly pipeline and statistics are shown in Supplementary Data 4 , respectively. Both CEGMA ${ }^{38}$ and BUSCO v2 ${ }^{39}$ were used to evaluate the completeness of assembled genome. Fosmid libraries were prepared following the manual of CopyControl Fosmid Library Production Kit (Epicentre, Madison, WI). In addition, 74 publicly available E. crus-galli EST sequences and 156,757 transcripts generated by this study were aligned to the assembled genome by $\mathrm{GMAP}^{40}$ for validation. For E. oryzicola line ZJU2 (collected from $23^{\circ} 31^{\prime} \mathrm{N}, 111^{\circ}$ $\left.43^{\prime} \mathrm{E}\right)$, paired-end DNA libraries $(800 \mathrm{bp}$ ) were prepared and sequenced by HiSeq2500. Clean reads were mapped to the assembled E. crus-galli genome by Bowtie $2^{41}$ and the mapping result was visualized using inGAP ${ }^{42}$.

Genome gene and repeat annotation. We constructed an E. crus-galli repeat library using RepeatModeler v1.0.5 ${ }^{43}$ with the default parameters. Two complementary programs (RECON and RepeatScout) were configured in RepeatModeler and were used for identification of repeat family sequences in the genome. The resulting E. crus-galli repeat library was further used to run RepeatMasker v3.1.2 ${ }^{43}$ for the whole genome repeat annotation.

A hybrid strategy combining ab initio predictions, homologous gene evidence and transcriptomic support (RNA-seq) was applied in gene prediction. Three ab initio gene finders, GeneMark.hmm ${ }^{44}$, Fgenesh ${ }^{45}$, and Augustus ${ }^{46}$ were used. Protein sequences of three closely related species (Z. mays, S. bicolor, S. italica) were aligned to the assembled E. crus-galli genome using Spaln $2^{47}$ to get evidences of the gene structure. RNA-seq data generated from the whole plant of E. crus-galli were used for gene annotation. The reads were de novo assembled by Trinity ${ }^{48}$ into raw transcripts. The Seqclean utility implemented in PASA ${ }^{49}$ was applied to 
identify evidence of polyadenylation, strip the poly-As, trim vectors, and to discard low-quality sequences. The remaining transcripts were aligned to the E. crus-galli genome using GMAP. The valid transcript alignments were clustered and further assembled by PASA based on genomic location and then the transcriptome-based consensus gene models were generated. All gene structures predicted by the above procedures were integrated into consensus gene models by $\mathrm{EVM}^{50}$. The predicted genes were then checked manually. The final set of genes were determined using the following criteria: (1) Partial gene models without start or stop codons were removed; (2) No 'N's residing in the coding sequences (CDS); (3) Sequences homologous with the Repbase (E-value $\leq 1 \mathrm{e}-5$, identity $\geq 30 \%$, coverage $\geq 30 \%$ and minimum matching length $\geq 30$ aa) were removed.

The predicted E. crus-galli genes were aligned against non-redundant green plant protein databases in Swiss-Prot and NR protein databases for functional annotation (BLASTP, E-value $\leq 1 \mathrm{e}-5$ ). Protein domains were predicted by comparing the sequences against various domain databases, including Pfam, TIGRFAM, ProDom, and SMART using InterProScan v $5^{51}$. Gene ontology (GO) terms for each gene were assigned based on corresponding InterPro entries. Noncoding RNAs were predicted by the Infernal program using default parameters ${ }^{52}$.

Phylogenetic tree and genomic synteny. We identified paralogs and orthologs using OrthoMCL v1.4 with the default settings (BLASTP E-value $\leq 1 \mathrm{e}-5$ and MCL inflation parameter of 1.5$)^{53}$. The protein sequence sets from six sequenced plants (B. distachyon, O. sativa, S. bicolor, S. italica, Z. mays, A. thaliana; Phytozome v9.0) and E. crus-galli were used to perform gene family identification. The protein sequences of single-copy genes among the seven species identified by OrthoMCL were aligned using MAFFT ${ }^{54}$. After the Gblocks ${ }^{55}$ alignment optimization, singlecopy genes with aligned protein sequences longer than 1000 amino acids (aa) were chosen for phylogenetic tree construction and divergence time estimation. Species divergence times were estimated using an uncorrelated relaxed clock in BEAST v1.7.5 with A. thaliana as an outgroup ${ }^{56}$. Monophyletic constraints were imposed for the nodes that were used to calibrate the evolutionary rates (Blosum62 and an uncorrelated exponential relaxed model). We used a Yule speciation process, which specifies a constant rate of species divergence. Normal priors were used for monocot-dicot split time (mean: 150.0 Mya, std dev: 4.0) and for O. sativa-Z. mays split time (mean: $50.0 \mathrm{Mya}$, std dev: 4.0). The MCMC chains in BEAST were run for 10,000,000 generation sampling every 1,000 steps. Convergence between the runs and the amount of burn-in (throwing away some iterations at the beginning of an MCMC run) was determined using Tracer v1. $5^{57}$, which was used to assess the effective sample size and to check the consistency of the results. The tree was drawn with FigTree ${ }^{58}$. For genomic synteny, we first used BLASTP (E-value $\leq 10-7$ ) to align the E. crus-galli protein dataset to that of S. bicolor, S. italica and itself. The alignments were then subjected to DAGchainer ${ }^{59}$ to determine syntenic blocks. The $4 \mathrm{dTv}$ of each syntenic block was calculated by an in-house Perl script.

To build phylogenetic trees for the genes of momilactone A gene clusters, ten grass species (including $O$. sativa, O. punctata, O. brachyantha, Zizania latifolia, Brachypodium distachyon, S. bicolor, Z. mays, Panicum virgatum, and Setaria italic, E. crus-galli) were involved and Arabidopsis thaliana was used as outgroup. BLASTP was used to scan homologous genes to rice genes of the cluster in the protein dataset (E-value thresholds: MAS: 1e-80; KSL4, CPS4, and CYP99A2: 1e100). Raxml v8 $8^{60}$ was applied with the parameters '-m PROTGAMMAAUTO -auto-prot $=$ bic' to automatically select the best protein model for tree construction ${ }^{60}$. Each tree was constructed with 100 bootstraps.

Investigations for P450 and GST. Members of the CYP450 (PF00067) and GST (PF13417, PF13410, PF13409, PF00043, and PF02798) gene families were identified by InterProScan $v 5^{51}$. The phylogenetic tree was constructed by Fasttree ${ }^{61}$ with protein sequence alignments by MAFFT v7. $2^{54}$. For divergence estimation of CYP450 and GST genes, phylogenetic trees of CYP450 and GST genes in E. crus-galli and O. sativa were constructed by Fasttree ${ }^{61}$ with an Arabidopsis gene (CYP450: AT1G75130.1; GST: AT1G59670.1) as outgroup, respectively. The substitution distances are measured by MEGA v5.262. On the basis of identified Pfam information for all genes of five grass species (O. sativa, B. distachyon, S. bicolor, S. italica, Z. mays) and E. crus-galli based on InterProScan $5^{51}$, enrichment analysis was conducted to examine whether CYP450 and GST genes are over-presented in the E. crus-galli genome by hypergeometric test.

Genomic and transcriptomic investigations for allelopathy. We searched barnyardgrass orthologs in known biosynthesis gene clusters ${ }^{15}$ to identify candidate gene clusters in the E. crus-galli genome that are involved in the allelopathic interaction.

The relay seeding in agar (RSA) method ${ }^{63}$ with a few modifications was used to investigate the allelopathic interactions between barnyardgrass (STB08) and rice (PI312777). Ten germinated PI312777 seeds were first transferred to a plastic tissue culture box ( $10 \mathrm{~cm}$ in base diameter) filled with $50 \mathrm{ml}$ of medium containing $0.5 \%$ agar, and arranged in three rows with a 3-4-3 pattern (Supplementary Fig. 5). Germinated STB08 seeds were first transferred to a Petri dish with sterile water. Five days later, 10 germinated STB08 seeds were transferred to the tissue culture box containing germinated PI312777 seeds with a pattern of five STB08 seeds between two rows of PI312777 seeds (Supplementary Fig. 5). The PI312777 and STB08 seedlings were co-cultured in a SAFE incubator (Ningbo, China) kept at
75\% relative humidity. Mono-cultured STB08 seedlings (i.e., seedlings growing alone, under the same conditions) were used as controls. The entire co- and monocultured STB08 seedlings were collected at $3 \mathrm{~h}$ and RNA extracted for RNA-Seq analysis. A total of three biological replicates were collected for each condition.

Illumina RNA-Seq libraries were prepared and sequenced on a

HiSeq4000 system following the manufacturer's instructions. The raw paired-end reads were first filtered into clean data using NGSQCtookit v2.3.3 with default settings ${ }^{64}$. The cleaned reads were then aligned to the E. crus-galli genome assembly using Tophat v2.0.965. After alignment, the count of mapped reads from each sample was derived and normalized to fragments per kilobase of exon per million fragments mapped for each predicted transcript using the Cufflinks package $^{66}$. The resulting alignment files were then supplied to Cuffdiff in the Cufflinks package for differential expression analysis.

The allelopathic transcriptomic profile of our experiment was investigated using the Mapman software ${ }^{67}$. All E. crus-galli genes were first submitted to the online functional annotation sever called Mercator ${ }^{68}$. The $\log 2$ ratio of fold change (coculture/mono-culture) was calculated for each significantly differentially expressed gene. The resulting files were loaded into Mapman to visualize the transcriptomic profile and perform the pathway enrichment study.

Experiments for DIMBOA measurement. For DIMBOA measurement, mono(six plants in a tissue culture box) or co-cultivated (three Echinochloa and three rice plants in a box) hydroponic Echinochloa weeds (STB08) and rice (PI312777) seedlings at the first-leaf stage were transplanted to deionized water for $45 \mathrm{~min}, 1.5$ and $3 \mathrm{~h}$. Both mono- and co-cultivated Echinochloa and rice plants (whole plant) were then analyzed by HPLC after freeze-drying.

Experiments for $\boldsymbol{P}$. oryzae infection. Pyricularia oryzae strains were cultured on agar plates containing complete medium for 10 days and conidia were harvested and re-suspended in $0.2 \%(\mathrm{w} / \mathrm{v})$ gelatin solution. About $10^{5}$ conidia per $\mathrm{ml}$ were sprayed evenly onto E. crus-galli plants at the three-leaf stage using an artist's airbrush (Badger Co., Illinois) with the $0.2 \%(\mathrm{w} / \mathrm{v})$ gelatin solution as control ${ }^{69}$. The inoculated plants were placed in a dew chamber at $22^{\circ} \mathrm{C}$ for $48 \mathrm{~h}$ in the dark and then transferred in a growth chamber under a photoperiod of $12 \mathrm{~h}$ for 7 days. Leaves with lesion was used for examination of gene expression by qRT-PCR. The relative value for the expression level of MAS and KSL4 genes was calculated by the $2^{-\Delta \Delta C_{\mathrm{T}}}$ method using the Tubulin gene as an internal control. Primers for qRTPCR were given in Supplementary Table 7.

Data availability. All raw DNA sequencing reads (Illumina and PacBio data) for $E$. crus-galli de novo assembly, sequencing data for E. oryzicola, and RNA-Seq data for the gene prediction and allelopathic experiments have been deposited under NCBI BioProject PRJNA268892. The SRA accession numbers are SRR5920284SRR5920293, SRR5902661, SRR5903813-SRR5903830, and SRR5903559SRR5903564. The E. crus-galli genome assembly and the annotated genes are accessible at http://ibi.zju.edu.cn/RiceWeedomes/Echinochloa/, and the assembly is also available at ENA (European Nucleotide Archive) under assembly accession GCA_900205405. All relevant data contained within the paper are available from the corresponding author on request.

Received: 15 December 2016 Accepted: 16 August 2017 Published online: 18 October 2017

\section{References}

1. Lundkvist, A. \& Verwijst, T. (ed. Nokkoul, R.) Research in Organic Farming 157-186 (InTech, 2011)

2. Vigueira, C. C., Olsen, K. M. \& Caicedo, A. L. The red queen in the corn: agricultural weeds as models of rapid adaptive evolution. Heredity 110, 303-311 (2013).

3. Warwick, S. I. \& Stewart, C. N. Crop Ferality and Volunteerism 9-30 (CRC, 2005).

4. Michael, T. P. \& VanBuren, R. Progress, challenges and the future of crop genomes. Curr. Opin. Plant. Biol. 24, 71-81 (2015).

5. Peng, Y. et al. De novo genome assembly of the economically important weed horseweed using integrated data from multiple sequencing platforms. Plant. Physiol. 166, 1241-1254 (2014).

6. Bajwa, A. A. et al. Eco-biology and management of Echinochloa crus-galli. Crop. Prot. 75, 151-162 (2015).

7. Kraehmer, H., Jabran, K., Mennan, H. \& Chauhan, B. S. Global distribution of rice weeds A review. Crop. Prot. 80, 73-86 (2016).

8. Aoki, D. \& Yamaguchi, H. Genetic relationship between Echinochloa crus-galli and Echinochloa oryzicola accessions inferred from internal transcribed spacer and chloroplast DNA sequences. Weed Biol. Manag 8, 233-242 (2008). 
9. Yabuno, T. Biosystematic study of the genus Echinochloa. Jpn J. Bot. 19, 277-323 (1966).

10. Barrett, S. C. H. Crop Mimicry in Weeds. Econ. Bot. 37, 255-282 (1983).

11. Norsworthy, J. K., Wilson, M. J., Scott, R. C. \& Gbur, E. E. Herbicidal activity on acetolactate synthase-resistant barnyardgrass (Echinochloa crus-galli) in Arkansas, USA. Weed Biol. Manag. 14, 50-58 (2014).

12. Oerke, E. C. \& Dehne, H. W. Safeguarding production-losses in major crops and the role of crop protection. Crop. Prot. 23, 275-285 (2004).

13. Jabran, K., Mahajan, G., Sardana, V. \& Chauhan, B. S. Allelopathy for weed control in agricultural systems. Crop. Prot. 72, 57-65 (2015).

14. Khanh, T., Xuan, T. \& Chung, I. Rice allelopathy and the possibility for weed management. Ann. Appl. Biol. 151, 325-339 (2007).

15. Boycheva, S., Daviet, L., Wolfender, J. \& Fitzpatrick, T. B. The rise of operon-like gene clusters in plants. Trends Plant. Sci. 19, 447-459 (2014).

16. Frey, M., Schullehner, K., Dick, R., Fiesselmann, A. \& Gierl, A. Benzoxazinoid biosynthesis, a model for evolution of secondary metabolic pathways in plants. Phytochemistry. 70, 1645-1651 (2009).

17. Shimura, K. et al. Identification of a biosynthetic gene cluster in rice for momilactones. J. Biol. Chem. 282, 34013-34018 (2007).

18. Swaminathan, S., Morrone, D., Wang, Q., Fulton, D. B. \& Peters, R. J. CYP76M7 is an ent-cassadiene C11 $\alpha$-hydroxylase defining a second multifunctional diterpenoid biosynthetic gene cluster in rice. Plant. Cell. 21, 3315-3325 (2009).

19. Yu, Q. \& Powles, S. Metabolism-based herbicide resistance and cross-resistance in crop weeds: A threat to herbicide sustainability and global crop production. Plant. Physiol. 166, 1106-1118 (2014).

20. Dilday, R. H., Mattice, J. D., Moldenhauer, K. A. \& Yan, W. Allelopathic potential in rice germplasm against ducksalad, redstem and barnyard grass. J. Crop Prot. 4, 287-301 (2001).

21. Vogt, T. Phenylpropanoid biosynthesis. Mol. Plant 3, 2-20 (2010)

22. Bartwal, A., Mall, R., Lohani, P., Guru, S. K. \& Arora, S. Role of secondary metabolites and brassinosteroids in plant defense against environmental stresses. J. Plant. Growth. Regul. 32, 216-232 (2013).

23. Sue, M., Nakamura, C. \& Nomura, T. Dispersed benzoxazinone gene cluster: molecular characterization and chromosomal localization of glucosyltransferase and glucosidase genes in wheat and rye. Plant. Physiol. 157, 985-997 (2011)

24. Kato-Noguchi, H. \& Peters, R. J. The role of momilactones in rice allelopathy. J. Chem. Ecol. 39, 175-185 (2013).

25. Hasegawa, M. et al. Phytoalexin accumulation in the interaction between rice and the blast fungus. Mol. Plant-Microbe Interact 23, 1000-1011 (2010).

26. Miyamoto, K. et al. Evolutionary trajectory of phytoalexin biosynthetic gene clusters in rice. Plant. J. 87, 293-304 (2016).

27. Ming, R. \& Wai, C. M. Assembling allopolyploid genomes: no longer formidable. Genome. Biol. 16, 27 (2015).

28. Sierro, N. et al. The tobacco genome sequence and its comparison with those of tomato and potato. Nat. Commun. 5, 3833 (2014).

29. Zhang, T. et al. Sequencing of allotetraploid cotton (Gossypium hirsutum L. acc. TM-1) provides a resource for fiber improvement. Nat. Biotechnol. 33, 531-537 (2015)

30. Chalhoub, B. et al. Early allopolyploid evolution in the post-Neolithic Brassica napus oilseed genome. Science 345, 950-953 (2014).

31. Takos, A. M. \& Rook, F. Why biosynthetic genes for chemical defense compounds cluster. Trends Plant. Sci. 17, 383-388 (2012).

32. Song, L., Florea, L. \& Langmead, B. Lighter: fast and memory-efficient sequencing error correction without counting. Genome. Biol. 15, 509 (2014).

33. Luo, R. et al. SOAPdenovo2: an empirically improved memory-efficient shortread de novo assembler. Gigascience 1, 18 (2012).

34. Gao, S., Bertrand, D., Chia, B. K. H. \& Nagarajan, N. OPERA-LG: efficient and exact scaffolding of large, repeat-rich eukaryotic genomes with performance guarantees. Genome. Biol. 17, 102 (2016).

35. Pryszcz, L. P. \& Gabaldon, T. Redundans: an assembly pipeline for highly heterozygous genomes. Nucleic Acids. Res. 44, e113 (2016).

36. Koren, S. et al. Canu: scalable and accurate long-read assembly via adaptive k-mer weighting and repeat separation. Genome Res. 27, 722-736 (2017).

37. English, A. C. et al. Mind the gap: upgrading genomes with Pacific Biosciences RS long-read sequencing technology. PLoS ONE 7, e47768 (2012).

38. Parra, G., Bradnam, K. \& Korf, I. CEGMA: a pipeline to accurately annotate core genes in eukaryotic genomes. Bioinformatics 23, 1061-1067 (2007).
39. Simao, F. A., Waterhouse, R. M., Ioannidis, P., Kriventseva, E. V. \& Zdobnov, E. M. BUSCO: assessing genome assembly and annotation completeness with singlecopy orthologs. Bioinformatics 31, 3210-3212 (2015).

40. Wu, T. D. \& Watanabe, C. K. GMAP: a genomic mapping and alignment program for mRNA and EST sequences. Bioinformatics 21, 1859-1875 (2005).

41. Langmead, B. \& Salzberg, S. L. Fast gapped-read alignment with Bowtie 2. Nat. Methods 9, 357-359 (2012).

42. Qi, J., Zhao, F., Buboltz, A. \& Schuster, S. C. inGAP: an integrated next-generation genome analysis pipeline. Bioinformatics 26, 127-129 (2009).

43. Tarailo-Graovac, M. \& Chen, N. Using RepeatMasker to identify repetitive elements in genomic sequences. Curr. Protoc. Bioinformatics Chapter 4, Unit $4.10(2009)$

44. Lukashin, A. V. \& Borodovsky, M. GeneMark.hmm: new solutions for gene finding. Nucleic. Acids. Res. 26, 1107-1115 (1998).

45. Salamov, A. A. \& Solovyev, V. V. Ab initio gene finding in Drosophila genomic DNA. Genome Res. 10, 516-522 (2000).

46. Stanke, M. et al. AUGUSTUS: ab initio prediction of alternative transcripts. Nucleic. Acids. Res. 34, W435-W439 (2006).

47. Iwata, H. \& Gotoh, O. Benchmarking spliced alignment programs including Spaln2, an extended version of Spaln that incorporates additional speciesspecific features. Nucleic Acids Res. 40, e161 (2012).

48. Grabherr, M. G. et al. Full-length transcriptome assembly from RNA-Seq data without a reference genome. Nat. Biotechnol. 29, 644-652 (2011).

49. Haas, B. J. et al. Improving the Arabidopsis genome annotation using maximal transcript alignment assemblies. Nucleic. Acids. Res. 31, 5654-5666 (2003).

50. Haas, B. J. et al. Automated eukaryotic gene structure annotation using evidence modeler and the program to assemble spliced alignments. Genome. Biol. 9, R7 (2008).

51. Zdobnov, E. M. \& Apweiler, R. InterProScan-an integration platform for the signature-recognition methods in InterPro. Bioinformatics 17, 847-848 (2001).

52. Nawrocki, E. P. \& Eddy, S. R. Infernal 1.1: 100-fold faster RNA homology searches. Bioinformatics 29, 2933-2935 (2013).

53. Li, L., Stoeckert, C. J. \& Roos, D. S. OrthoMCL: identification of ortholog groups for eukaryotic genomes. Genome Res. 13, 2178-2189 (2003).

54. Katoh, K., Misawa, K., Kuma, K. i. \& Miyata, T. MAFFT: a novel method for rapid multiple sequence alignment based on fast Fourier transform. Nucleic Acids Res. 30, 3059-3066 (2002).

55. Castresana, J. Selection of conserved blocks from multiple alignments for their use in phylogenetic analysis. Mol. Biol. Evol. 17, 540-552 (2000).

56. Drummond, A. J., Suchard, M. A., Xie, D. \& Rambaut, A. Bayesian phylogenetics with BEAUti and the BEAST 1.7. Mol. Biol. Evol. 29, 1969-1973 (2012).

57. Rambaut, A. \& Drummond, A. J. Tracer v1.5. http://beast.bio.ed.ac.uk/Tracer (2009)

58. Rambaut, A. FigTree 1.3, http://tree.bio.ed.ac.uk/software/figtree/ (2009)

59. Haas, B. J., Delcher, A. L., Wortman, J. R. \& Salzberg, S. L. DAGchainer: a tool for mining segmental genome duplications and synteny. Bioinformatics 20, 3643-3646 (2004).

60. Stamatakis, A. RAxML version 8: a tool for phylogenetic analysis and post-analysis of large phylogenies. Bioinformatics. 30, 1312-1313 (2014).

61. Price, M. N., Dehal, P. S. \& Arkin, A. P. FastTree: computing large minimum evolution trees with profiles instead of a distance matrix. Mol. Biol. Evol. 26, 1641-1650 (2009).

62. Tamura, K. et al. MEGA5: Molecular evolutionary genetics analysis using maximum likelihood, evolutionary distance, and maximum parsimony methods. Mol. Biol. Evol. 28, 2731-2739 (2011).

63. Navarez, D. \& Olofsdotter, M. (ed. Brown, H.) Proceedings of 1996 Second International Weed Control Congress 1285-1290 (Copenhagen, Denmark: DJF Flakkebjerg, 1996).

64. Patel, R. K. \& Jain, M. NGS QC Toolkit: a toolkit for quality control of next generation sequencing data. PLoS ONE 7, e30619 (2012).

65. Trapnell, C., Pachter, L. \& Salzberg, S. L. TopHat: discovering splice junctions with RNA-Seq. Bioinformatics 25, 1105-1111 (2009).

66. Trapnell, C. et al. Transcript assembly and quantification by RNA-seq reveals unannotated transcripts and isoform switching during cell differentiation. Nat. Biotechnol. 28, 511-515 (2010).

67. Thimm, O. et al. MAPMAN: A user-driven tool to display genomics data sets onto diagrams of metabolic pathways and other biological processes. Plant. J. 37, 914-939 (2004) 
68. Lohse, M. et al. Mercator: a fast and simple web server for genome scale functional annotation of plant sequence data. Plant. Cell Environ. 37, 1250-1258 (2014).

69. Su, Z. Z. et al. Evidence for biotrophic lifestyle and biocontrol potential of dark septate endophyte harpophora oryzae to rice blast disease. PLOS ONE 8, e61332 (2013).

\section{Acknowledgements}

This work was financed by State Key Lab of Rice Biology of China, Zhejiang Natural Science Foundation (LZ17C130001), National Natural Science Foundation of China (31401453), the Fundamental Research Funds for the Central Universities (16+X Project),Jiangsu Collaborative Innovation Center for Modern Crop Production, the 111 Project (B17039), and China Postdoctoral Science Foundation (2017M612002). We gratefully acknowledge the support of the IBM high performance computing cluster of Analysis Center of Agrobiology and Environmental Sciences, Zhejiang University.

\section{Author contributions}

L.F. and L.G. designed the research. H.Y., D.-S.K., D.A., L.G., Y.-Y.W., G.L., F.F. C.L., Q.S., C.-Y.Y. and L.F. collected and identified the Echinochloa species. J.Q., G.J., H.Z., Y.-F.W. and W.S. sequenced and assembled the genomes. J.Q., G.J., L.M., H.Z., L.J., Z.L., L.C., E.S., W.W., Q.C., D.W., S.W., Q.P., Y.Z., X.Z., L.W., L.W. and C.-Y.Y. performed sequence analysis. C.-H.K., H.Y., T.Y., X.Y., E.Y., K.M. and K.O. measured the content of DIMBOA. C.Z. and C.X. performed the P. oryzae experiment. Y.L., H.Y., D.C., C.-H.K., L.G., M.T., Q.Q. and L.B. discussed the data. L.F., J.Q. and M.T. wrote the paper.

\section{Additional information}

Supplementary Information accompanies this paper at doi:10.1038/s41467-017-01067-5.

Competing interests: The authors declare no competing financial interests.

Reprints and permission information is available online at http://npg.nature.com/ reprintsandpermissions/

Publisher's note: Springer Nature remains neutral with regard to jurisdictional claims in published maps and institutional affiliations.

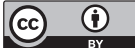

Open Access This article is licensed under a Creative Commons Attribution 4.0 International License, which permits use, sharing, adaptation, distribution and reproduction in any medium or format, as long as you give appropriate credit to the original author(s) and the source, provide a link to the Creative Commons license, and indicate if changes were made. The images or other third party material in this article are included in the article's Creative Commons license, unless indicated otherwise in a credit line to the material. If material is not included in the article's Creative Commons license and your intended use is not permitted by statutory regulation or exceeds the permitted use, you will need to obtain permission directly from the copyright holder. To view a copy of this license, visit http://creativecommons.org/ licenses/by/4.0/.

(C) The Author(s) 2017 